InnOvaciOnes de NegOciOs 8(16): 335-356, 2011

(c) 2011 UANL, Impreso en México (ISSN: 2007-1191)

\title{
El uso del método MICMAC y MACTOR análisis prospectivo en un área operativa para la búsqueda de la excelencia operativa a través del Lean Manufacturing (Use of the MICMAC and MACTOR method prospective analysis in an operational area for the pursuit of operational excellence through the Lean Manufacturing)
}

\author{
Juan Baldemar Garza Villegas ^ \& Dante Vladimir Cortez Alejandro* \\ "Universidad Autónoma de Nuevo León, San Nicolás de los Garza, N.L., México. \\ *Universidad Autónoma de Coahuila, Saltillo, Coah., México. \\ Email: juan.garzavs@uanl.edu.mx
}

Keywords: Lean Manufacturing, MICMAC, MACTOR, prospective, TQM,

Abstract. Presents the MICMAC and MACTOR prospective analysis study in an operational area which looks for world-class management with reference to the concepts of lean manufacturing. The results of a qualitative analysis and his conclusions are presented.

Palabras clave: MICMAC, MACTOR, prospectiva, ATQ, Manufactura Esbelta.

Resumen. Se presenta el estudio de análisis prospectivo MICMAC y MACTOR en un área operativa que busca una gestión de clase mundial haciendo referencia a los conceptos de la manufactura esbelta. Se presentan los resultados de un análisis cualitativo prospectivo y sus conclusiones.

\section{Introducción}

La Prospectiva como método cualitativo en la industria de la manufactura en México se pudiera considerar como un tema nuevo e innovador. Su termino etimológico se deriva del verbo en latín prospectare 0 prospicere que significa pro, "adelante" y spectare, que significa mirar mejor. En resumen significaría "mirar mejor y mas lejos aquello que esta por venir" 
Existen solo algunos estudios que se han desarrollado en nuestro país. Anaya (2010). La gran mayoría enfocado a estudios sociales y de políticas públicas, estudios de universidades y/o estudios del sector de salud.

La OCDE define la prospectiva como: el conjunto de "tentativas sistemáticas para observar a largo plazo el futuro de la ciencia, la tecnología, la economía y la sociedad con el propósito de identificar las tecnologías o métodos emergentes que probablemente produzcan los mayores beneficios económicos y/o sociales".

El análisis estructural es el método cualitativo de la prospectiva y lo pudiéramos definir como una reflexión colectiva relacionando diferentes elementos de un sistema con la perspectiva de provocar el cambio en el futuro. La prospectiva posee herramientas metodológicas que facilitan y sistematizan la reflexión colectiva sobre el futuro y la construcción de imágenes o escenarios de futuro. Godet (2001).

\section{Materiales y método}

El método estructural MICMAC busca analizar de manera cualitativa las relaciones entre las variables que componen un sistema dentro de una empresa, organización, sociedad, país etc. Como se mencionó anteriormente es parte del análisis estructural y se apoya en el juicio cualitativo de actores y/o expertos que son parte de un sistema. El acrónimo MICMAC proviene de las palabras: Matriz de Impactos Cruzados Multiplicación Aplicada a una Clasificación método elaborado por M. Godet en colaboración con J.C. Duperrin de acuerdo a Godet (2007, p. 65). El objetivo del Análisis Estructural MICMAC es identificar las principales variables, influyentes y dependientes; así como las variables esenciales para la evolución del sistema.

Las diferentes fases del método MICMAC de acuerdo a Godet (1997) son las siguientes:

Fase 1: listado de las variables del sistema,

Fase 2: la descripción de relaciones entre variables del sistema, y

Fase 3: la identificación de variables clave y sus categorías e interpretación. 


\section{Perfil de los Actores y/o Expertos.}

En el caso particular de este proceso de validez de contenido de esta investigación el perfil de los actores y/o expertos consideró lo siguiente: Experto académico ó práctico de manufactura esbelta y ser empleado de la empresa en estudio. Las personas que fueron elegidas no sólo debieron ser grandes conocedores del área sobre la que se realizó el estudio, sino que presentaron una pluralidad en sus planteamientos. Esta pluralidad fue necesaria para evitar la aparición de sesgos en la información disponible.

En resumen los 10 participantes cubrieron el perfil requerido para la prueba de prospectiva. 4 participantes fueron del área de producción, 2 de ingeniería, 2 de calidad y 2 de mejora continua. El llenado fue cualitativo y participaron los diferentes actores del sistema, este proceso de evaluación se realizó los días 10 y 11 de Agosto del 2011. Por cada pareja de variables, se planteó la pregunta siguiente: ¿existe una relación de influencia directa entre la variable i y la variable j? si es que no, anotamos 0 , en el caso contrario, nos preguntamos si esta relación de influencia directa es, débil (1), mediana (2), fuerte (3) o potencial (P) de acuerdo a Godet (2007, p.64).

En la Figura 1 se muestra la captura en el software MICMAC de las variables de estudio: Desempeño de Procesos, Capacidad de los Integradores (recursos), Reducción del Desperdicio, Control Visual, Balanceo y Secuenciación (Control de la Producción), Planeación de la Demanda, Identificación de Flujos de Valor, Sistema de Calidad - Compartir las Mejores Prácticas, Sistema de Calidad - Actividades, Sistema de Calidad Integradores, Mejora Continua, TPM, Administración de Tableros de Desempeño, Planeación Estratégica Gerencial, Estandarización, Salud, Seguridad y Medio Ambiente, 5'S y Liderazgo. Todas estas variables son parte de un sistema nombrado Sistema Lean Manufacturing para la excelencia operativa en esta empresa privada.

El análisis MICMAC provee una matriz y un gráfico nombrado Plano de Influencia y Dependencia de las variables del sistema de estudio y las categoriza en: Variables de entorno, variables reguladoras, palancas secundarias, variables objetivo, variables clave, variables resultado, variables autónomas y variables determinantes de acuerdo a Godet (2007) 
Figura 1. Lista de variables del estudio de investigación

\begin{tabular}{|c|c|c|}
\hline $\begin{array}{l}\text { Desempeño de } \\
\text { Procesos }\end{array}$ & Des Proc & Se refiere a la capacidad del proceso \\
\hline $\begin{array}{l}\text { Capacidad de los } \\
\text { Integradores }\end{array}$ & Cap Int & $\begin{array}{l}\text { Se refiere a la capacidad de los integradores } \\
\text { (Recursos) para el desempeño de los procesos }\end{array}$ \\
\hline $\begin{array}{l}\text { Red. del } \\
\text { Desperdicio }\end{array}$ & Red. Desp & $\begin{array}{l}\text { Se refiere a la reducción del desperdicio } \\
\text { (Rechazos y Reprocesos) } \\
\text { Busca incementar el Flujo. }\end{array}$ \\
\hline Control Visual & CVisual & $\begin{array}{l}\text { Se refiere al control visual del área para dar } \\
\text { flujo a la operación. }\end{array}$ \\
\hline Bal y Sec & Bal y Sec & $\begin{array}{l}\text { Se refiere al balanceo y la secuenciación del } \\
\text { proceso. Busca incrementar el flujo operativo. }\end{array}$ \\
\hline $\begin{array}{l}\text { Plan de la } \\
\text { Demanda }\end{array}$ & Plan Dem & $\begin{array}{l}\text { Se refiere a la planeación de la demanda. } \\
\text { Busca incrementar flujo operativo del proceso. }\end{array}$ \\
\hline $\begin{array}{l}\text { Identificación de } \\
\text { Flujos de Valor }\end{array}$ & Id.Flu Val & $\begin{array}{l}\text { Es la capacidad de indentificar la generación de } \\
\text { valor en el flujo operativo. }\end{array}$ \\
\hline Sistema de Calidad & Sis Cal & $\begin{array}{l}\text { Se refiere al sistema de calidad que cuenta el } \\
\text { proceso para su control operativo. }\end{array}$ \\
\hline $\begin{array}{l}\text { Actividades Sist de } \\
\text { Calidad }\end{array}$ & Act SCal & $\begin{array}{l}\text { Se refiere a las actividades específicas del } \\
\text { sistema de calidad. }\end{array}$ \\
\hline $\begin{array}{l}\text { Sistema de Calidad } \\
\text { Integradores }\end{array}$ & SisCallnte & $\begin{array}{l}\text { Se refiere a los integradores del sistema de } \\
\text { calidad y su capacidad de dar control } \\
\text { operativo. }\end{array}$ \\
\hline Mejora Continua & $\mathrm{MC}$ & Se refiere al sistema de Mejora continua. \\
\hline TPM & TPM & Se refiere al sistema de TPM. \\
\hline $\begin{array}{l}\text { Tableros de } \\
\text { Desempeño }\end{array}$ & $\begin{array}{l}\text { Tab de } \\
\text { Des }\end{array}$ & $\begin{array}{l}\text { Se refiere a la medición básica del desempeño } \\
\text { a través de un scorecard. }\end{array}$ \\
\hline $\begin{array}{l}\text { Planeación } \\
\text { Estratégica }\end{array}$ & Plan Est & Se refiere al proceso de planeación estratégica. \\
\hline Estandarización & Estandariz & $\begin{array}{l}\text { Se refiere a la estandarización de las prácticas } \\
\text { operativas. }\end{array}$ \\
\hline $\begin{array}{l}\text { Salud, Seguridad y } \\
\text { Medio Ambiente }\end{array}$ & SSA & $\begin{array}{l}\text { Se refiere a la promoción de la salud, seguridad } \\
\text { y cuidado del medio ambiente del área. }\end{array}$ \\
\hline 5's & 5 's & Se refiere a las 5's. \\
\hline Liderazgo & Lid & Se refiere al Liderazgo de los mandos. \\
\hline
\end{tabular}

Fuente. Elaboración propia en software LIPSOR-EPITA-MICMAC.

\section{J.B. Garza \& D.V. Cortez}




\section{Alcances y Limitaciones}

La interpretación del gráfico Plano de Influencia y Dependencia permite una lectura completa del sistema según resulten ser las variables motrices 0 dependientes. Es decir, para cualquier variable su valor estratégico estaría determinado por la suma de su valor de motricidad y de su valor de dependencia. $E n=m n+d n$. Godet (1997).

La combinación de ambos resultados es la que definitivamente define a las variables según su tipología. Su disposición en el plano en relación a las diagonales nos ofrece una primera clasificación, tal y como queda reflejado en la Figura 2.

Figura 2. Plano de Influencia y Dependencia del estudio

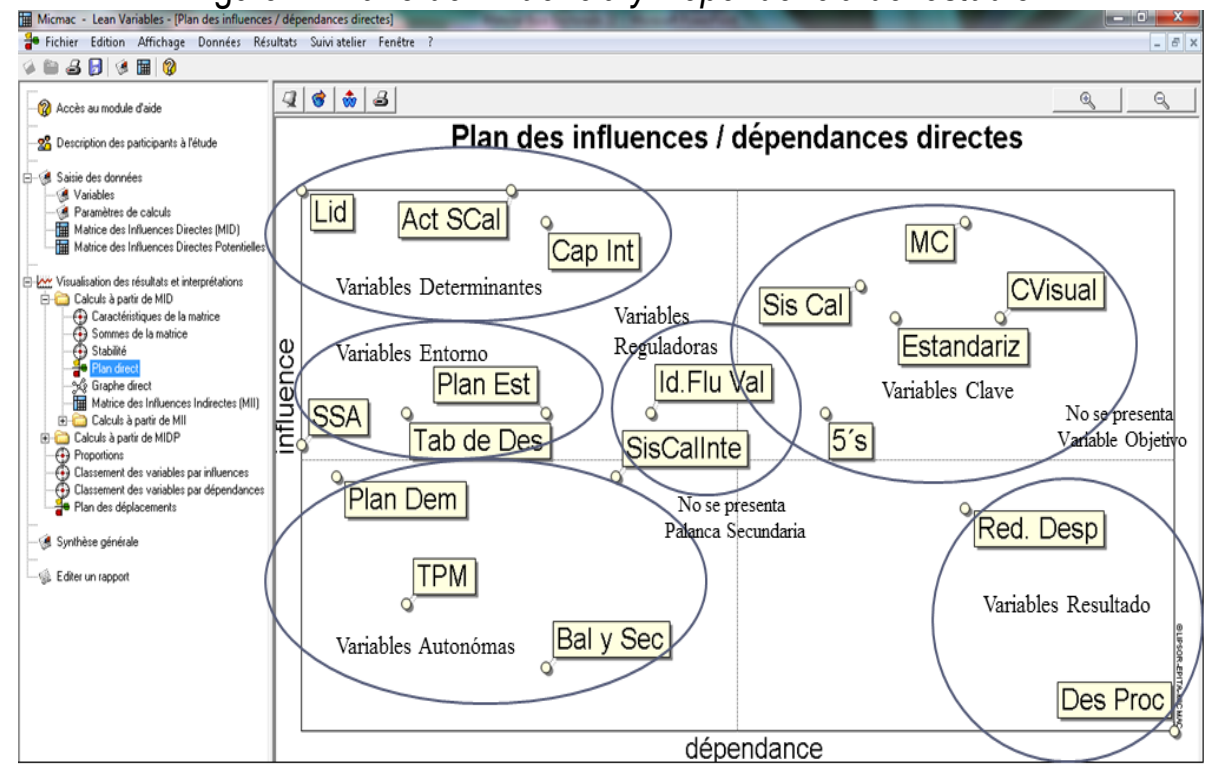

Fuente. Elaboración propia en software LIPSOR-EPITA-MICMAC.

Aquí en la Figura 2 podemos observar 6 categorías (círculos) de variables formadas de acuerdo al resultado que proporcionó el software del análisis MICMAC. En en el siguiente apartado de resultados se describe de manera detallada la reflexión del análisis en el estudio de investigación de acuerdo a las categorías definidas por el método MICMAC Godet (2001) y sus supuestos. 


\section{Resultados}

Variables Determinantes Se encuentran en la zona superior izquierda del plano de influencia y dependencia, son las variables que según su evolución a lo largo del periodo de estudio se convierte en frenos o motores del sistema. Estas pudieran ser propulsoras o inhibidoras del sistema. El objetivo es que sean propulsoras y determinen las conductas adecuadas del sistema.

- Liderazgo (Liderazgo de Mandos)

- Act S Cal (Actividades del Sistema de Calidad)

- Cap de Integradores (Capacidad de Integradores)

Variables Entorno Son las variables con escasa dependencia del sistema pueden ser consideradas un decorado del sistema, se encuentran en la zona media de la parte izquierda del plano de influencia y dependencia. El objetivo es complementar su valor agregado al sistema.

- SSA (Seguridad, Salud y Medio Ambiente)

- Plan Est (Planeación Estratégica)

- Tab de Des (Tablero de Desempeño)

Variables Reguladoras Son las variables situadas en la zona central del plano de influencia y dependencia se convierten en llave de paso para alcanzar el cumplimiento de las variables clave. Determinan el buen funcionamiento del sistema en condiciones normales. Se sugiere evaluar de manera consistente y con frecuencia periódica estas variables.

- Id. Flujo de Valor (Identificación del Flujo de Valor)

- SisCallnte (Sistema de Calidad y sus integradores)

Palancas Secundarias Son las variables complementarias a las variables reguladoras, actuar sobre ellas significa hacer evolucionar las variables reguladoras que a su vez afectan a la evolución de las variables clave. En el plano de influencia y dependencia se encuentran ubicadas debajo de las reguladoras.

-No se presentaron en el estudio de investigación.

Variables Objetivo Son las variables que se ubican en la parte central a la derecha en el plano de influencia y dependencia. Son muy dependientes y medianamente motrices, de ahí su carácter de tratamiento como objetivos, 
puesto que en ellas se puede influir para que su evolución sea aquella que se desea

-No se presentaron en el estudio de investigación.

Variables Clave Son las variables que se encuentran en la zona superior derecha del plano de influencia y dependencia tambien nombradas variables reto del sistema. Son muy motrices y muy dependientes, perturban el funcionamiento normal del sistema, estas variables sobredeterminan el propio sistema. Son por naturaleza inestables y se corresponden con los retos del sistema. En resumen deben continuamente tener retos que propicien el cambio del sistema a un nivel más optimo. Son las que mantienen lubricado el sistema.

-MC (Mejora Continua)

-Sis Cal (Sistema de Calidad)

-5 's (5's)

-Estandariz (Estandarización)

-CVisual (Control Visual)

Variables Resultado Son variables que se caracterizan por su baja motricidad y alta dependencia se encuentran en la zona inferior derecha del plano de influencia y dependencia, y suelen ser junto con las variables objetivo, indicadores descriptivos de la evolución del sistema. Se trata de variables que no se pueden abordar de frente sino a través de las que dependen en el sistema. Estas variables requieren un seguimiento y monitoreo estrecho que permita verificar la efectividad del sistema en general.

-Red. Desp (Reducción de Desperdicio)

-Des Proc (Desempeño de los Procesos)

Variables Autonómas Son variables poco influyentes o motrices y poco dependientes, se corresponden con tendencias pasadas 0 inercias del sistema o bien están desconectadas de él. En el plano de influencia y dependencia se encuentran en la zona inferior izquiera. No constituyen parte determinante para el futuro del sistema. Se constata frecuentemente un gran número de acciones de comunicación alrededor de estas variables que no constituyen un reto. Hay que alinear estas variables a la estrategia Lean de la 
empresa. Se sugiere dar más valor a esas variables. En este momento son poco influyentes en el sistema.

-Plan Dem (Planeación de la Demanda)

-TPM (Total Productive Maintenance / Mantenimiento Productivo Total)

-Bal y Sec (Balanceo y Secuenciación)

\section{Materiales y método}

El método MACTOR (Método, Actores, Objetivos, Resultados de Fuerza) busca valorar las relaciones de fuerza entre los actores y estudiar sus convergencias y divergencias con respecto a un cierto número de posturas y de objetivos asociados. Las diferentes fases del método MACTOR según Godet (1990) son las siguientes:

Fase 1: Identificar los actores que controlan o influyen sobre las variables clave del análisis estructural: listado de actores.

Fase 2: Identificar los objetivos estratégicos de los actores respecto a las variables clave: listado de objetivos.

Fase 3: Evaluar las influencias directas entre los actores: jerarquización de actores mediante un cuadro de influencias entre actores (MAA o Matriz de Actores x Actores).

4: el actor Ai puede cuestionar la existencia del actor $\mathrm{Aj}$

3: el actor Ai puede cuestionar las misiones del actor $A j$

2: el actor Ai puede cuestionar los proyectos del actor $\mathrm{Aj}$

1: el actor Ai puede cuestionar, de manera limitada (durante algún tiempo o en algún caso concreto) la operativa del actor Aj.

0 : el actor Ai no tiene ninguna influencia sobre el actor $\mathrm{Aj}$

Fase 4: Conocer el posicionamiento de los actores respecto a los objetivos. Describir la actitud actual de cada actor respecto a cada objetivo (opuesto, neutro, indiferente o favorable). Representación matricial Actores $x$ Objetivos.

Signo positivo: el actor es favorable al objetivo.

Signo negativo: el actor es desfavorable al objetivo.

Punto 0: el actor, es neutro cara al objetivo

\section{J.B. Garza \& D.V. Cortez}




\section{Ponderado}

4: el objetivo cuestiona la existencia del actor o es imprescindible para la existencia del actor;

3: el objetivo cuestiona el cumplimiento de las misiones del actor o es imprescindible a sus misiones;

2: el objetivo cuestiona el éxito de los proyectos del actor o es imprescindible para estos proyectos;

1: el objetivo cuestiona, de una forma limitada en el tiempo y espacio los procesos operativos (gestión, etc.....) del actor 0 es imprescindible para estos procesos operativos.

0: el objetivo tiene poca o ninguna incidencia.

Fase 5: Conocer el grado de convergencia y de divergencia entre los actores y el plano de la distancia que existe entre los diferentes objetivos del sistema. http://www.3ie.fr/lipsor/lipsor_es/mactor_es.html

Figura 3. Fase 1 MACTOR. Lista de actores principales para la búsqueda de la excelencia operativa Lean Manufacturing. Ver perfil de actores

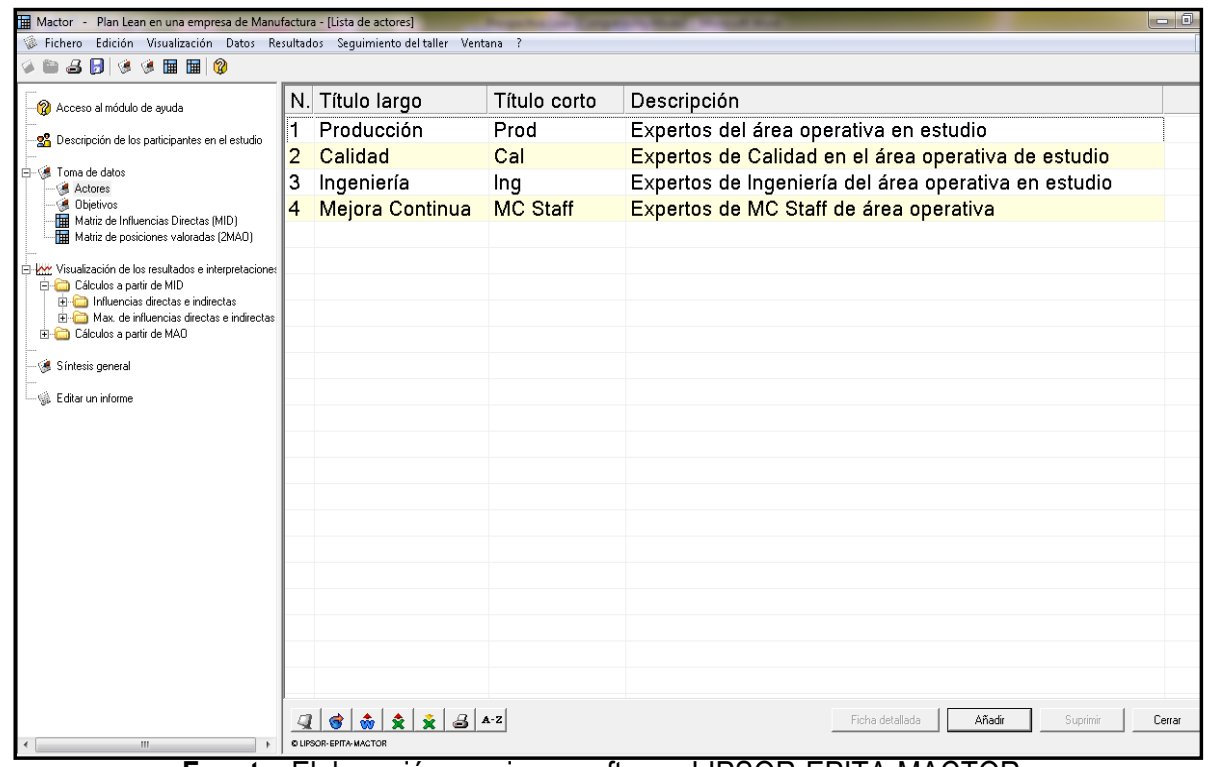

Fuente. Elaboración propia en software LIPSOR-EPITA-MACTOR. 
Como se puede apreciar en la Figura 3 los actores principales fueron personas de las áreas de producción, ingeniería, calidad y de mejora continua. $Y$ en la Figura 4 se enlistan los objetivos organizacionales de la empresa que resultaron de la planeación estratégica y que fueron punto de partida para el análisis MACTOR. El consenso de esos expertos de cada área involucrada en el sistema se expreso de manera numérica en el software MACTOR.

Figura 4. Fase 2 MACTOR. Lista de Objetivos para la búsqueda de la excelencia operativa a través del Lean Manufacturing

\begin{tabular}{|c|c|c|c|}
\hline \multirow{2}{*}{\multicolumn{4}{|c|}{ 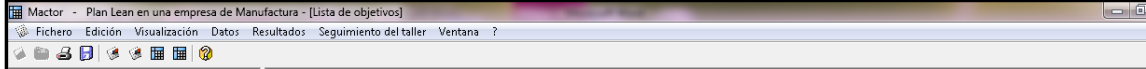 }} \\
\hline & & & \\
\hline \multirow{6}{*}{ 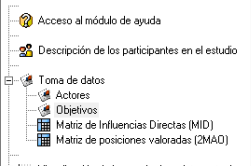 } & Título largo & Título corto & Descripción \\
\hline & Asegurar tiempo de Entrega & Tiem Ent & Asegurar el tiempo de entrega del producto \\
\hline & Costo de Mantenimiento & Costo Mtto & Asegurar un costo optimo de Mtto en el área operative \\
\hline & Mejorar Productividad & Productiv & Incrementar la productividad del área operativa \\
\hline & Reducción de Costos & Red Costos & Reducir el costo del proceso. \\
\hline & Reducción del Lead Time & Lead Time & Reducir el tiempo de conducción \\
\hline \multirow{4}{*}{ 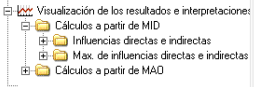 } & Reducción de Desperdicio... & Red Desp & Reducir desperdicios. Calidad en el Proceso \\
\hline & Reducir Reprocesos & Red. Rep & Reducir los Reprocesos \\
\hline & Tiempo de Respuesta en & Resp Cot & Tiempo de respuesta en cotizaciones. \\
\hline & Producción & Prod & Se refiere a la producción \\
\hline & & & \\
\hline
\end{tabular}

Fuente. Elaboración propia en software LIPSOR-EPITA-MACTOR.

\section{Materiales y método}

En esta fase 2 MACTOR se listan los objetivos para la búsqueda de la excelencia operativa Lean Manufacturing: En esta fase se detallaron nueve objetivos: Asegurar tiempo de entrega, optimizar costo de mantenimiento, mejorar productividad, reducir costos, reducir lead time, reducir desperdicios, reducir reprocesos, tiempo de respuesta en cotizar el producto y producción "el día a día". Estos objetivos de la figura 4 fueron definidos en base a la planeación estratégica de la empresa que se realizó el día 19 de Septiembre del 2011.

La fase 3 MACTOR. Determina la influencia que un actor A ejerce sobre un actor $B$ y esta la llama influencia directa. Si ejerce esta influencia sobre un actor $\mathrm{C}$ el cual él mismo influye sobre el actor $\mathrm{B}$, se tratará de una influencia indirecta. El programa MACTOR mide las influencias y dependencias directas e indirectas mediante indicadores sumando los términos de la matriz MIDI ( $i$ y $D i$, respectivamente).

\section{J.B. Garza \& D.V. Cortez}


La matriz MIDI permite obtener las influencias directas e indirectas de orden 2 entre actores. El interés de esta matriz es el de aportar una visión más completa del juego de relaciones de fuerza (un actor puede limitar el abanico de elección de un segundo actuando sobre él mismo a través de un actor relevo). En el caso particular de esta investigación la matriz de orden 2 coincide en términos generales con la matriz de orden 1 .

Mediante la MIDI se calculan dos indicadores:

El grado de influencia directa e indirecta de cada actor ( $I i$, sumando por líneas). El grado de dependencia directa e indirecta de cada actor (Di, sumando por columnas).

Elementos de cálculo:

La Matriz de Influencias Directas e Indirectas $(M I D I)$ se calcula de la manera siguiente: $(M I D I)_{i j}=(M I D)_{i j}+\sum k \operatorname{Min}\left((M I D)_{i k},(M I D)_{k j}\right)$.

En el segundo término de la ecuación, "(MIDI)ij expresa la influencia directa que el actor $i$ ejerce sobre el actor $j$ y " $\sum k$ Min $\left((M I D)_{i k},(M I D)_{k j}\right.$ " representa la suma de todas las influencias indirectas que el actor $i$ ejerce sobre el actor $j$ y que pasan por un actor relevo $k$. Para éste último valor, sólo se tienen en cuenta influencias indirectas de orden 2, es decir, influencias que transitan sólo por un actor relevo cada vez. Las influencias indirectas de orden 3, de orden 4 etc (transitan por 2, 3 actores relevo, etc... antes de llegar al actor $j$ ) no se tienen en cuenta. Se considera que un actor $i$ que desee influir indirectamente sobre un actor $j$ no puede integrar en sus cálculos las decenas, incluso centenares de influencias indirectas que se alternan por varios actores formando una cadena. Por el contrario, este actor puede ejercer varias influencias indirectas de orden 2 , cada una de ellas transitando por un sólo actor relevo a la vez.

La influencia directa e indirecta neta del actor $i(I i)$ se calcula sumando las influencias que este actor tiene sobre los otros actores, es decir, sin tener en cuenta las influencias indirectas que pueda tener sobre él mismo:

$I i=\sum k_{i}^{1}(M I D I)_{i k}$. La dependencia directa e indirecta neta del actor $i(D i)$ se calcula sumando las influencias que este actor recibe de otros actores, es decir, sin tener en cuenta las influencias indirectas que él pueda recibir de él

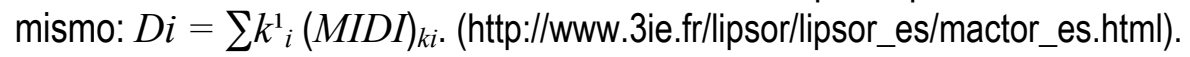




\section{Resultados}

Aquí en la Figura 5 podemos observar que las áreas de producción e ingeniería tienen una alta influencia y dependencia en el sistema se encuentran en el cuadrante superior derecho según Godet (2007) son los actores de enlace del sistema. Es en esta zona del cuadrante superior derecho en donde se puede presentar el conflicto. Es importante realizar acuerdos que logren maximizar los resultados esperados. El área de calidad tiene poca influencia y mediana dependencia se encuentra en el cuadrante inferior derecho conocido según Godet (2007) como el actor dominado.

Figura 5.- Fase 4 MACTOR. Plano de Influencias y dependencias entre los actores del sistema

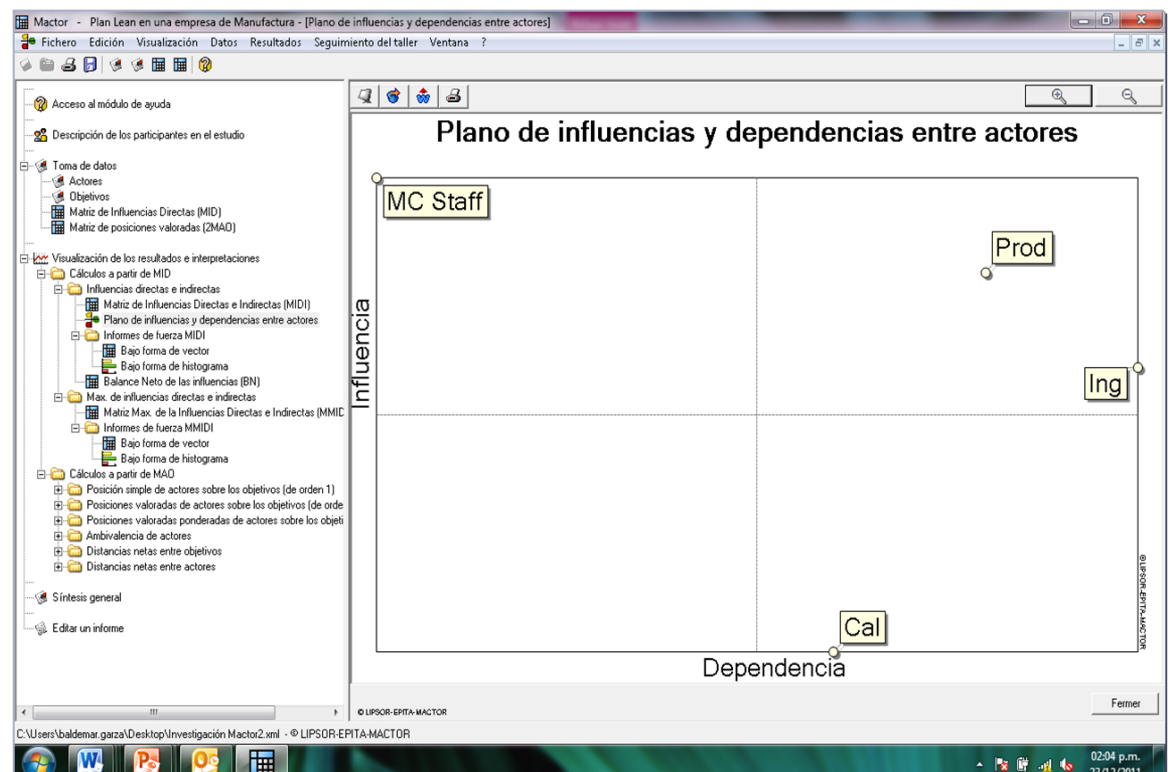

Fuente. Elaboración propia en software LIPSOR-EPITA-MACTOR.

El área de mejora continua tiene alta influencia pero nula dependencia en el sistema. Es decir el área de mejora es muy independiente. Prácticamente el área de mejora continua se encuentra en la Zona de Poder que es el cuadrante superior izquierdo esta área según Godet (2007) es el actor dominante. Aquí es importante dosificar esta posible fuerza que pueda

\section{J.B. Garza \& D.V. Cortez}


ejercer esta área en el resto de las áreas. Una posible interpretación de lo anterior sugiere que es necesario buscar integrar más la parte de calidad y buscar desarrollar la dependencia del área de mejora continua con las áreas de producción, ingeniería y calidad. Las decisiones deberán ser en consenso. No aparecieron actores en el cuadrante inferior izquierdo según Godet (2007) actores autónomos 0 aislados.

Aquí en la figura 6 podemos observar que las áreas de producción e ingeniería convergen fuertemente, luego le sigue el departamento de mejora continua y por último se puede apreciar poca convergencia del departamento de calidad. La convergencia en términos simples sugiere la coincidencia de ideas, tendencias e intereses entre los diferentes actores del sistema. Como se mencionó anteriormente, en el caso particular de esta investigación la matriz de orden 2 coincide en términos generales con la matriz de orden 1. Es por eso que no se anexo el plano de convergencias entre actores de orden 2. Los cambios no fueron significativos. Prácticamente se obtuvo el mismo resultado.

Figura 6. Fase 5 MACTOR. Plano de Convergencias entre los actores del sistema

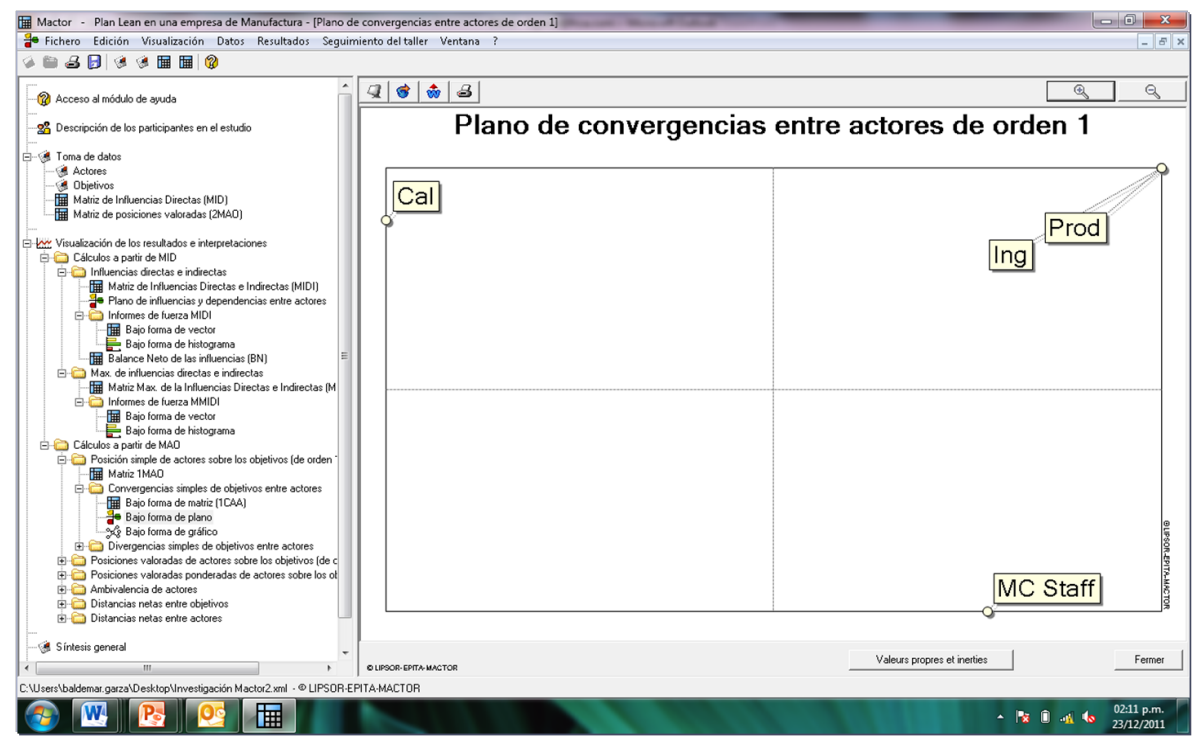

Fuente. Elaboración propia en software LIPSOR-EPITA-MACTOR.

MICMAC/MACTOR \& Manufactura Esbelta 
El plano de distancias netas entre objetivos se presenta en la Figura 7 este plano nos permite obtener los objetivos sobre los cuales los actores están posicionados de la misma manera (en acuerdo o en desacuerdo). Este plano sirve para separar grupos de objetivos sobre los cuales los actores están en fuerte convergencia (cuando los objetivos están cerca los unos de los otros) o en fuerte divergencia (cuando los objetivos están lejos los unos de los otros).

Este plano posiciona los objetivos sobre un mapa en función de la balanza neta obtenida por diferencia entre la Matriz valorada de convergencias y aquella de divergencias de objetivos. Aquí podemos observar que el objetivo de producción lo que se conoce coloquialmente como el "día a día" esta muy alejado a los otros objetivos provocando distracción en estos y no logrando las metas de mediano y largo plazo. Algunos especialistas sugieren que el modelo de producción contemple el presente o corto plazo, el mediano y largo plazo.

Figura 7. Fase 5 MACTOR. Plano de distancias entre los actores del sistema

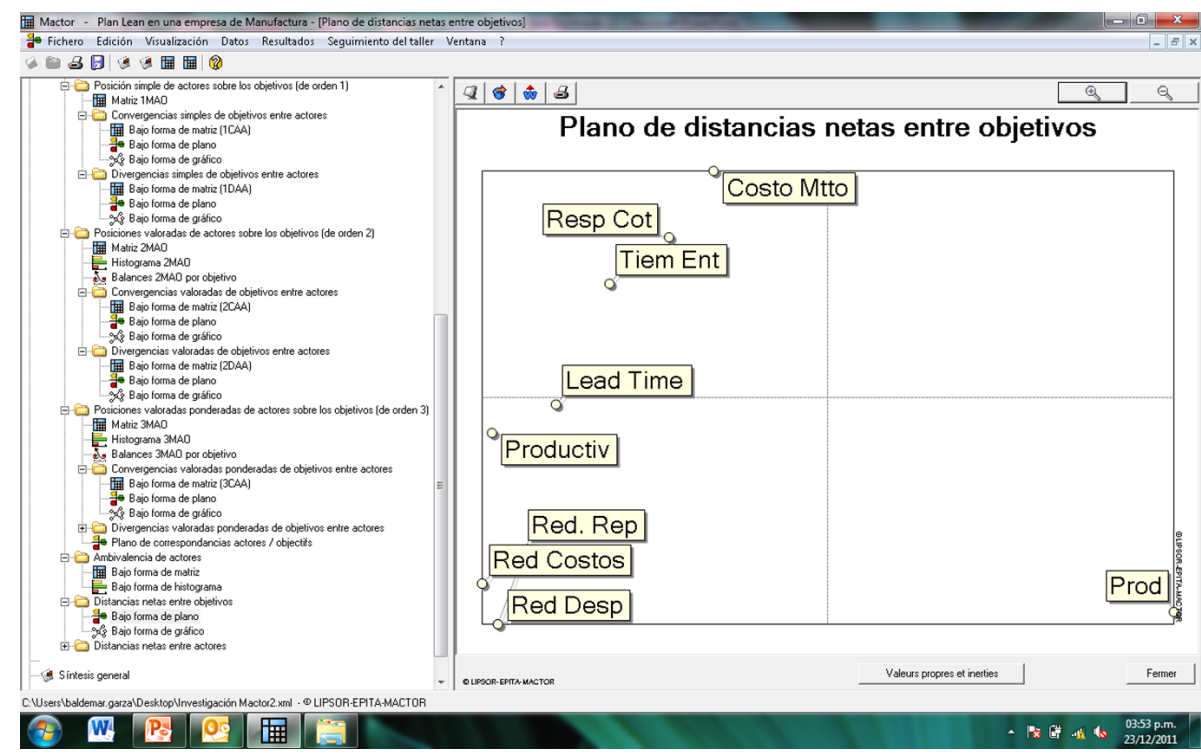

Fuente. Elaboración propia en software LIPSOR-EPITA-MACTOR.

J.B. Garza \& D.V. Cortez 


\section{Materiales y método}

El modelo Lean de la empresa se basa en 18 criterios que evalúan la excelencia a través de 80 subcriterios en una escala nominal de 1 a 5 . Cada subcriterio se encuentra definido de manera detallada y se cuenta con un glosario que permite el pleno entendimiento del subcriterio que se evalúa. En la Figura 8 se presenta modelo conceptual.

Escala de 1 a 5 nominal del modelo Lean y su significado:

1.- Incapaz de cumplir el subcriterio. No hay sistema de trabajo.

2.- Incipiente nivel de cumplimiento del subcriterio. Hay un inicio o piloto del sistema de trabajo pero aun es incipiente.

3.- Se ha logrado un nivel básico de entendimiento del sistema de trabajo. Se muestra desarrollo, despliegue e implementación.

4.- El sistema de trabajo es competente muestra madurez y sostenimiento.

5.- El sistema de trabajo ha logrado la excelencia muestra mejora continua y es referencia de clase mundial en la industria.

Figura 8. Entramado de criterios del modelo Lean

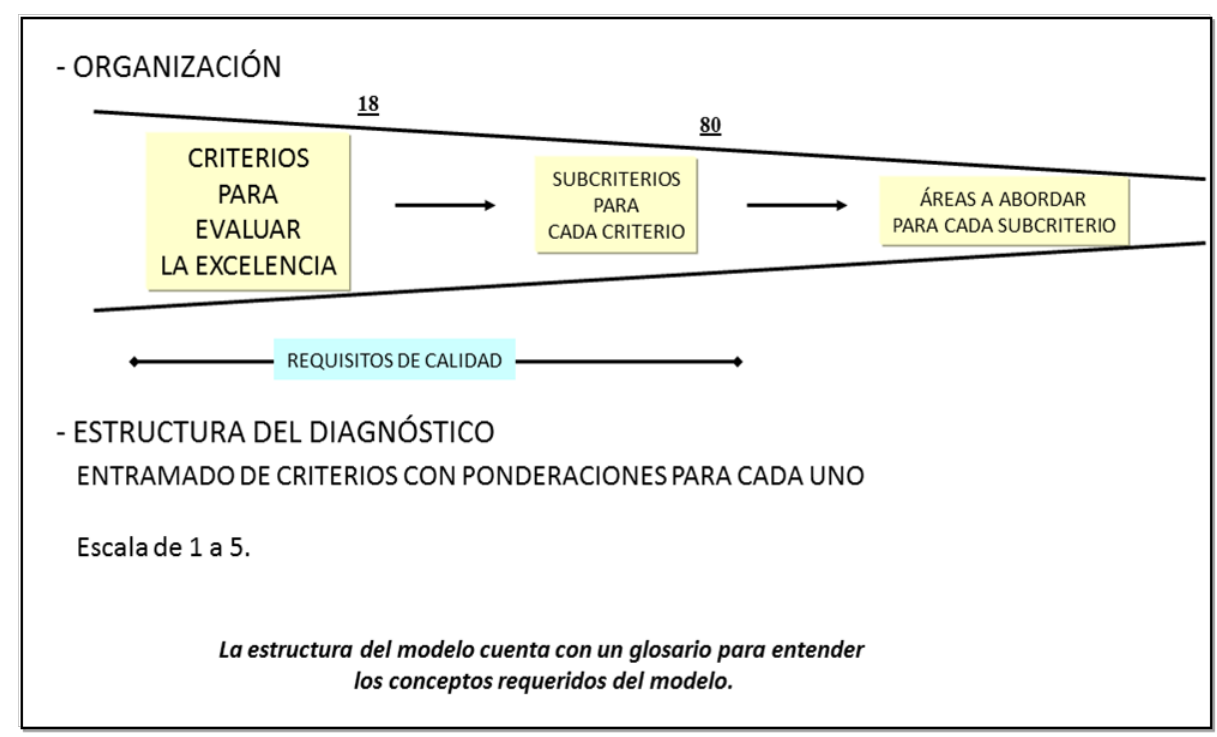

Fuente. Elaboración propia.

MICMAC/MACTOR \& Manufactura Esbelta 


\section{Alcances y Limitaciones}

Para la evaluación del modelo Lean en el área operativa se llevó el siguiente proceso: Se utilizó la escala nominal discreta de 1 a 5 para la evaluación de cada subcriterio en la figura 9 se muestra un ejemplo. Cada miembro del grupo de expertos-actores valora cada subcriterio, adjudicándole una calificación según su importancia. Se calculó después la media de los valores asignados a cada uno de los problemas y se obtuvo la clasificación final. Es importante mencionar que los subcriterios están basados en el TPS de sus siglas en ingles Toyota Production System pero es un modelo de producción particular de esta empresa en estudio.

Figura 9.- Ejemplo de evaluación de un subcriterio

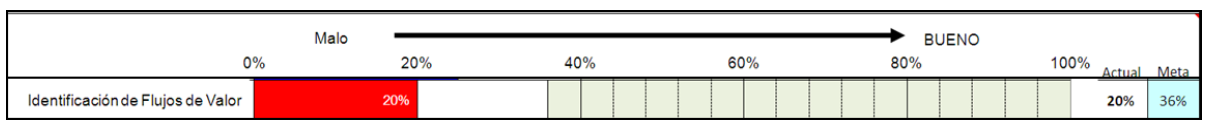

\begin{tabular}{|c|c|c|c|c|c|c|}
\hline \multicolumn{6}{|c|}{ Flujo del Proceso - Subcriterio.- Identificación de Flujos de Valor } & Cal \\
\hline \multirow[b]{2}{*}{$\begin{array}{l}\text { Atributo del } \\
\text { Proceso }\end{array}$} & 1 & 2 & 3 & 4 & 5 & \\
\hline & $\begin{array}{c}\text { No hay sistem a } \\
\text { o evidencia }\end{array}$ & Inicio/Piloto & $\begin{array}{c}\text { Desarrollol } \\
\text { Desplieguel } \\
\text { Im plem entación }\end{array}$ & $\begin{array}{l}\text { Maduraciónl } \\
\text { Sostenim iento }\end{array}$ & $\begin{array}{c}\text { Mejora } \\
\text { Continual Clase } \\
\text { Mundial }\end{array}$ & $\begin{array}{l}\text { Promedio } \\
\text { Consenso }\end{array}$ \\
\hline $\begin{array}{l}\text { Identificación } \\
\text { de los Flujos } \\
\text { de Valor }\end{array}$ & $\begin{array}{l}\text { El flujo de valor } \\
\text { no ha sido } \\
\text { definido por la } \\
\text { empresa. }\end{array}$ & $\begin{array}{c}\text { El flujo de valor } \\
\text { ha sido } \\
\text { identificado y } \\
\text { priorizado. Se } \\
\text { tiene un mapa } \\
\text { del estado } \\
\text { actual del flujo } \\
\text { de valor. }\end{array}$ & $\begin{array}{l}\text { Mapas de flujo } \\
\text { de valor del } \\
\text { estado actual y } \\
\text { futuro se } \\
\text { complementan } \\
\text { con planes de } \\
\text { acción para } \\
\text { evitar el } \\
\text { desperdicio. }\end{array}$ & $\begin{array}{l}\text { El estado futuro } \\
\text { ha sido } \\
\text { alcanzado con } \\
\text { mejoras } \\
\text { significantes en } \\
\text { el desempeño. }\end{array}$ & $\begin{array}{l}\text { El estado futuro } \\
\text { original ha sido } \\
\text { alcanzado. Se } \\
\text { añade un ciclo } \\
\text { de mejora } \\
\text { continua para } \\
\text { desafiar el } \\
\text { estado futuro } \\
\text { que está en } \\
\text { marcha. }\end{array}$ & 1 \\
\hline
\end{tabular}

Fuente. Elaboración propia.

Como se pude apreciar cada subcriterio tiene una descripción y nominación en cada nivel de la escala de 1 a 5 . Esto permitió evitar sesgo en el entendimiento de lo que se evalúa. La evaluación se realizó en una sala de juntas con apoyo de un moderador.

\section{J.B. Garza \& D.V. Cortez}




\section{Resultados}

A continuación en la Figura 10 Radar Lean se presenta el resumen de las calificaciones obtenidas por el grupo de actores-expertos.

Figura 10. Calificación del modelo Lean por actores-expertos y su meta

\section{Radar Lean Assesment}

Meta DActual

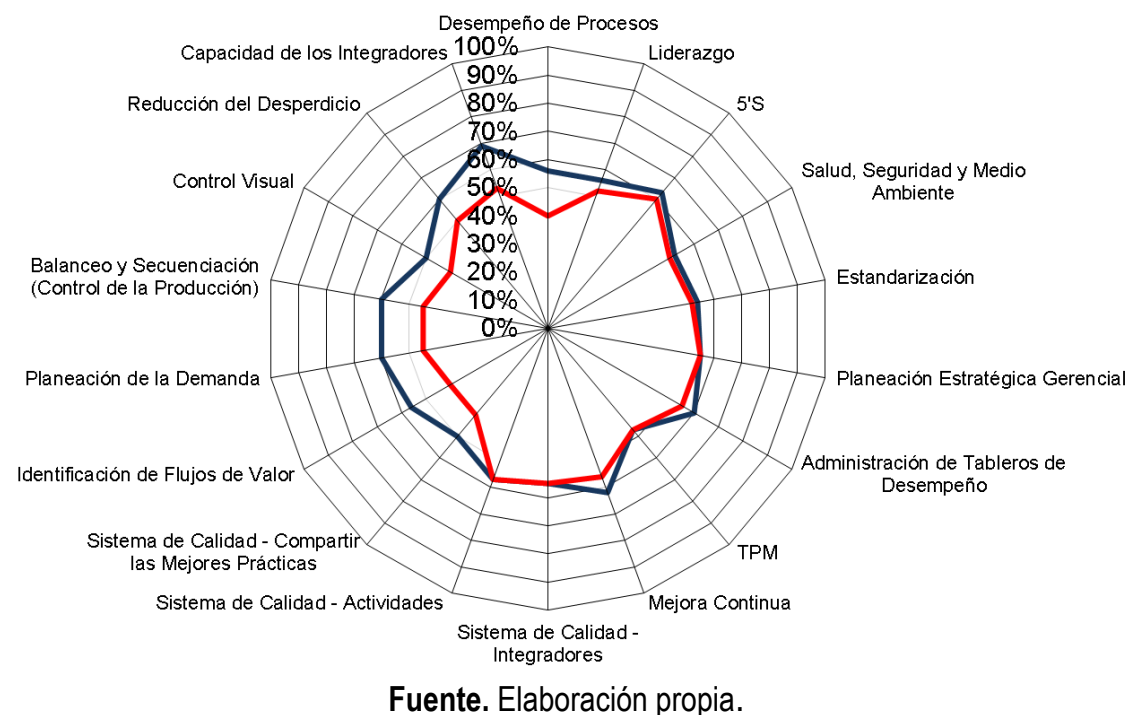

\section{Discusión}

El objetivo principal de este artículo fue presentar a la prospectiva como disciplina y como método, además de demostrar su validez y eficacia para generar información estratégica para la toma de decisiones, así como para el diseño, elaboración e implementación de actividades de mejora continua en los sistemas de trabajo. 
En este proceso de evaluación del método MICMAC y MACTOR, diez expertos fueron invitados para realizar el análisis prospectivo. Resultaron 6 categorías de variables formadas de acuerdo al resultado del análisis MICMAC del estudio y se describió de manera detallada la reflexión del análisis en el estudio de investigación de acuerdo a las categorías definidas por el método MICMAC. Godet (2001).

El método MACTOR tuvo como objetivo interesarse por los actores que de cerca o de lejos mandan señales sobre las variables claves para el futuro del sistema Lean y que surgieron del análisis estructural.

En este análisis se pudo observar que las áreas de producción e ingeniería convergen fuertemente, luego le sigue el departamento de mejora continua y por último se puede apreciar poca convergencia del departamento de calidad. La convergencia en términos simples sugiere la coincidencia de ideas, tendencias e intereses entre los diferentes actores del sistema sobre los objetivos específicos del sistema. Aquí se precisarán los roles y las responsabilidades de los diferentes actores del sistema. En el plano de distancias netas entre objetivos se posicionaron los objetivos sobre un mapping en función de la balanza neta obtenida por diferencia entre la matriz valorada de convergencias y aquella de divergencias de objetivos. Aquí se pudo observar que el objetivo de producción coloquialmente conocido como el "día a día" esta muy alejado a los otros objetivos de mediano y largo plazo provocando distracción en estos y no logrando las metas de mediano y largo plazo requeridas por la empresa.

Con respecto a las investigaciones futuras, es necesario estudiar la mejora continua en diferentes contextos y organizaciones. Los programas de prospectiva, como toda herramienta, requieren una capacidad de análisis importante por parte de los usuarios. Sin este análisis, una utilización demasiado mecánica del programa MICMAC y MACTOR puede enmascarar las verdaderas cuestiones y conduciría a contradicciones importantes 0 a falsas creencias.

Por otra parte otro objetivo del presente proyecto de investigación fue poder determinar el nivel de calificación de los 18 criterios que constituyen el Modelo de Lean Manufacturing creado por una empresa privada y los cuales resultaron relevantes de acuerdo a la opinión de los principales actores del proceso operativo de dicha empresa. Así mismo se busca analizar como dichos elementos se vuelven factores determinantes para la toma de

\section{J.B. Garza \& D.V. Cortez}


decisiones y de acuerdos; y como los mismos igualmente dificultan el proceso de adaptación a diversos procesos de excelencia de clase mundial.

Las herramientas y técnicas Lean con enfoque a proceso son sencillas de aprender y usar, el problema en las organizaciones es su implementación y sostenimiento. Fuera de Toyota el TPS es muchas veces conocido como Lean, Lean Manufacturing o Manufactura Esbelta, estas expresiones se han hecho populares en dos best sellers La máquina que cambio el mundo Womack, Jones, Roos (1991) y Lean thinking de Womack Jones (1996) ambos autores han dejado claro que la base es el Sistema de Producción Toyota es el Trabajo en Equipo.

Por ejemplo, Toyota referencia en Lean explícitamente enseña a las personas cómo mejorar su función operativa. No espera que estrictamente aprendan de la experiencia personal. (Spear, S. y Bowen, k. 2000). La regla del sistema de producción Toyota estipula que cualquier mejora en las actividades de producción debe realizarse de acuerdo con el método científico. Saber las causas que generan un problema y realizar acciones en consecuencia.

De acuerdo a Liker (2006) los especialistas pierden de vista los principios de gestión guía que rigen su Filosofía a Largo Plazo, el proceso correcto para producir resultados correctos, la capacidad para añadir valor a la organización mediante el desarrollo del personal, de sus socios y la resolución continua de los problemas fundamentales que impulsan el aprendizaje organizativo, de los cuales se desprenden una serie de comportamientos esperados en cada uno de ellos.

En términos generales la evaluación del modelo Lean resultó en una calificación promedio de 50 donde cada subcriterio se evaluó de 1 a 5 y la meta es lograr llegar a un nivel de 57 el siguiente año. Lo cual en términos cualitativos es lograr un entendimiento y desempeño pleno de los criterios y subcriterios de una empresa en vías de lograr la excelencia de clase mundial. En términos generales la empresa tiene un desempeño bueno sin embargo se busca un desempeño sobresaliente. Para lograr la meta anterior durante el mes de Noviembre y Diciembre se estableció un plan de trabajo que se le nombra cartera de proyectos con actividades específicas que permitan lograr el resultado. Dando seguimiento trimestral a lo anterior. Cada actividad 0 proyecto para lograr la meta del sistema lean esta alineada a un objetivo duro, que permita evaluar el grado de efectividad y estas actividades son 
parte del scorecard de desempeño de los actores del sistema. Se utilizará una clasificación para priorizar las actividades y/o proyectos del sistema Lean en: A, B y C. Donde los proyectos A serán los más relevantes y prioritarios.

Características de los proyectos A.

- Selección del proyecto por Jefatura del área y la Gerencia Operativa.

- Los proyectos A deben mostrar avance semanal a la Gerencia donde se da servicio. Se manda reporte semanal a Supervisor, Jefe y Gerente del área. (Sobre cumplimiento de variable "Y")

- Estos proyectos son coordinados principalmente por los actores principales: empleados del área de Mejora Continua y Jefes de las áreas operativas.

- Se mide el cumplimiento de la variable "Y" y su cumplimiento en el scorecard del área operativa.

- La idea es que se le de seguimiento a la variable "Y" cada semana.

- La idea es revisar estos proyectos "A" por lo menos una vez al mes con la Gerencia de Mejora Continua.

- Impacto económico superior a 15KUSD en el año.

A este plan de actividades 0 cartera de proyectos se le nombrará "Plan Lean" y a continuación se presenta de manera parcial un resumen del plan:

- 24 proyectos de piso enfocados a incrementar la eficiencia operativa.

- 4 Eventos tipo kaizen.

La palabra kaizen es una palabra japonesa que significa zen (buen) kai (cambio) kaizen = cambio por lo mejor. Un evento kaizen se enfocará a definir, describir los roles, e indicadores de los jefes, supervisores y apoyos del área operativa que se deberán gestionar. Otro evento se enfocará al TPM (Total Productive Maintenance / Mantenimiento Productivo Total) buscando reducir la demoras por fallas de equipo y lograr que la operación haga mantenimiento básico que permita detectar anomalías para su pronta corrección antes de que se conviertan en fallas. Otro evento se enfocará al set up (cambio de herramentales para cambio de producto) donde se enfocará a reducir el tiempo de cambio para lograr flexibilidad operativa y poder atender cualquier demanda de cualquier producto del cliente.

- 10 proyectos de Ingeniería enfocados a manejo de materiales.

- Proyecto de incorporación de equipos productivos etc 
Finalmente el análisis cualitativo MICMAC y MACTOR permitirán acciones específicas para lograr garantizar un buen despliegue de lo anterior y una buena convivencia y consenso entre los actores y objetivos definidos. El concepto es administrar de manera positiva el conflicto. Un ejemplo particular de una actividad específica para lo anterior será el poder reforzar el proceso de comunicación a través de un diplomado de "Trabajo en Equipo y Comunicación"

Por último las variables autónomas que resultaron del estudio MICMAC reflejan la importancia de cambiar a la brevedad, ya que se corre el riesgo de que se queden como conceptos aislados y sin valor agregado para el sistema en su conjunto. En el caso de la iniciativa TPM se estará evaluando ajustar las juntas semanales de productividad con un enfoque de más valor agregado. Esta petición fue sugerida por los expertos del área de producción. Otro ajuste que ya inició y sobre todo tiene mucha relación a la variable Plan Dem (Planeación de la Demanda) y Bal y Sec (Balanceo y Secuenciación) es que se estará evaluando la demanda por segmento a través de reuniones particulares con las UN (Unidades de Negocio). Cada UN tiene requerimientos específicos al área de producción es importante diferenciarlos y atenderlos de manera específica.

Es importante finalmente mencionar que la prospectiva no es un tema de planeación, no define las políticas y ni los objetivos. En resumen no remplaza los procesos de planeación y la toma de decisiones existentes, sino que complementa estas tres áreas, incrementando su efectividad, a partir de la generación de información estratégica para la toma de decisiones.

\section{Referencias}

Anaya, Lorena (2010). Propuesta Metodológica para de Diseño y Formulación de un Plan Estratégico Metropolitano Integral. El Caso de la Zona Metropolitana de Monterrey.

Godet M. (2001) Manual de prospectiva estratégica, Dunod, Paris: Dunod.

Godet, M. (1997). Manuel de Prospective Stratégique (Vol. 1). Paris: Dunod.

Godet, M. (1990) : El método MACTOR, Estratégico, revista de la Fundación para Estudios de la Defensa Nacional, Número de Junio.

Godet, M (2007). La Caja de Herramientas de la prospectiva estratégica. España. Laboratorio de investigación en prospectiva estratégica, CNAM, Paris, Instituto Europeo de Prospectiva estratégica. 
Laboratorio de Investigación en Prospectiva, Estrategia y Organización LIPSOR. http://www.laprospective.fr/

Liker, J.L. (2006). Las claves del éxito de Toyota: 14 principios de gestión del fabricante más grande del mundo, Barcelona: Gestión 2000

OCDE. Organización para la Cooperación y el Desarrollo Económico.

Spear, S. y Bowen, K. (2000). La decodificación del ADN del Sistema de Producción Toyota, Harvard Business Review. The President and Fellows of Harvard College.

Software MICMAC y MACTOR han sido desarrollados por el Instituto de Innovación Informática para la Empresa 31 E por petición del Laboratorio de Investigación en Prospectiva, Estrategia y Organización LIPSOR. http://www.3ie.fr/lipsor/lipsor_es/mactor_es.html

Womack, J.P, D.T. Jones \& D. Roos (1991).The machine that change the world: The story of lean production, New York: Harper Perennial.

Womack, J.P \& D.T. Jones (1996). Lean Thinking: banish waste and create wealth in your corporation, New York: Simon \& Schuster.

\section{J.B. Garza \& D.V. Cortez}

